\title{
Prevalência e fatores associados às quedas em idosos de Estação-RS: estudo transversal de base populacional
}

\author{
Prevalence and factors associated with falls in elderly from \\ Estação-RS: a cross-sectional population-based study
}

\author{
Alisson Padilha de Lima ${ }^{1,2}$, Ezequiel Vitório Lini², Marcos Paulo Dellani², \\ Marilene Rodrigues Portella², Marlene Doring ${ }^{2}$
}

\begin{abstract}
Resumo
Objetivo: Este estudo verificou os fatores associados às quedas em idosos de Estação, Rio Grande do Sul. Método: Estudo transversal com 418 idosos. No questionário foram contempladas as informações pessoais e familiares: sexo, faixa etária, cor, com quem reside, zona de moradia, estado marital e aposentadoria. Condições de saúde e hábito de vida como: dor crônica, alimenta-se sozinho, banha-se, deita/levanta da cama, sobe/desce escadas, artrite/artrose, dificuldade auditiva, dificuldade visual, hipertensão arterial, reumatismo, osteoporose e isquemia cerebral. A variável dependente foi "ocorrência de quedas nos últimos 12 meses que antecederam a entrevista”. Realizada frequência relativa e absoluta para característica da amostra. Testou-se a associação entre o desfecho e as variáveis independentes através do teste qui-quadrado, análises brutas e multivariada mediante regressão de Poisson, estimando-se as razões de prevalência brutas e ajustadas, calculados os respectivos intervalos de confiança de $95 \%$ a um $p \leq 0,050$. Resultados: A prevalência de quedas no ano anterior à pesquisa foi de $63,8 \%$. Após a análise múltipla permaneceram associadas às quedas as variáveis: analfabetismo $(R P=1,67)$, dor crônica $(R P=2,34)$ e isquemia cerebral $(R P=2,30)$. Conclusão: A investigação apresentou elevada prevalência de quedas entre os idosos e demonstra que os fatores associados às quedas são modificáveis e evitáveis.
\end{abstract}

Palavras-chave: acidentes por quedas; nível de saúde; envelhecimento da população.

\begin{abstract}
Objective: This study evaluated factors associated with falls in the elderly from Estação, Rio Grande do Sul. Method: A cross-sectional study with 418 elderly. Questionnaires included personal and family information: sex, age, race, who they live with, neighborhood, marital status and retirement. Health and life habit such as chronic pain, self-feeding, self-bathing, and get in/out of bed, up/down stairs, arthritis/arthrosis, hearing impairment, visual impairment, hypertension, rheumatism, osteoporosis and ischemia brain. The dependent variable was "occurrence of falls in the last 12 months preceding the interview." Calculated for relative and absolute frequency for sample characteristic. We tested the association between the outcome and independent variables through the chi-square test gross and multivariate analyzes using Poisson regression, estimating gross and adjusted prevalence ratios, calculated the confidence intervals of $95 \%$ to a $p \leq 0.05$. Results: The prevalence of falls in the year preceding the survey was $63.8 \%$. After multivariate analysis the following variables remained significantly associated: illiteracy $(P R=1,67)$, chronic pain $(P R=2,34)$ and cerebral ischemia $(P R=2,30)$. Conclusion: The research presented a high prevalence of falls among the elderly and shows that the associated factors are modifiable and preventable.

Keywords: accidental falls; health status; demographic aging.

${ }^{1}$ Faculdade de Educação Física da Associação Educacional Luterana BOM JESUS (IELUSC) - Joinville (SC), Brasil.

2Programa de Pós-Graduação Strictu Sensu em Envelhecimento Humano, Universidade de Passo Fundo (UPF/RS) - Passo Fundo (RS), Brasil.

Trabalho realizado na Saúde Pública de Estação - Rio Grande do Sul, Brasil.

Endereço para correspondência: Alisson Padilha de Lima - Faculdade de Educação Física Bom Jesus (IELUSC), Rua Mafra, 84 - Bairro Saguaçu -

CEP: 89221-665 - Joinville (SC), Brasil - Email: professor.alissonpadilha@gmail.com

Fonte de financiamento: CAPES/PROSUP.

Conflito de interesses: nada a declarar.
\end{abstract}

Este é um artigo publicado em acesso aberto (Open Access) sob a licença Creative Commons Attribution, que permite uso, distribuição e reprodução em qualquer meio, sem restrições desde que o trabalho original seja corretamente citado. 


\section{INTRODUÇÃO}

Os fatores associados e os índices de quedas estão entre as principais causas de morbidade e mortalidade por causas externas em adultos acima de 50 anos e são responsáveis por grande parte das incapacidades físicas decorrentes de lesões não intencionais, excluindo os acidentes de trânsito ${ }^{1}$. Ao longo da vida, na dimensão biológica, o processo de envelhecimento reduz as capacidades sensoriais e motores responsáveis pelo controle postural, resultando em um alto número de quedas entre os idosos ${ }^{2}$.

Modificações orgânicas de origem física e mental, como: diminuição das capacidades físicas e motoras, diminuição da capacidade funcional e cognitiva, aumento das doenças crônicas e psicológicas como as demências, estão associadas ao avanço da idade e fragilidade, aumentando a prevalência de lesões acometidas pelas quedas, pois todos os anos um terço dos idosos da comunidade correm o risco de cair, e cerca de 10 a 15\% suportam uma lesão ${ }^{3}$.

Estudos têm apontado diversos fatores de riscos para quedas em idosos, como: idade avançada, sexo feminino, baixo nível de escolaridade, fragilidade física, fraqueza muscular, marcha instável e equilíbrio, apresentar isquemia cerebral, cognição prejudicada e sintomas depressivos ${ }^{4,5}$.

A literatura exibe um entendimento de que o evento de quedas é um deslocamento não intencional do corpo para um nível inferior à posição inicial, um desequilíbrio postural com incapacidade de correção em tempo hábil ${ }^{6-9}$. Há um consenso de que a ocorrência das quedas em idosos se deve à participação de fatores intrínsecos, definidos como aqueles relacionados ao próprio sujeito, e extrínsecos os relacionados aos aspectos físicos do ambiente no qual o indivíduo interage ${ }^{10-12}$.

As quedas repercutem de modo significativo na vida dos caidores, portanto, as ações centradas na prevenção das quedas e também aquelas que intervêm nas repercussões de forma a dirimi-las ou evitá-las são necessárias para a maximização da capacidade funcional ${ }^{13}$.

Em vista da gravidade do problema decorrente das quedas na população idosa, tanto pela prevalência quanto pelos danos causados aos idosos, a ocorrência de quedas é um tema relevante e que necessita de constante pesquisa para a compreensão dos fatores causais e elaboração de novas ações preventivas por parte dos órgãos públicos de saúde. Diante desse contexto, este estudo teve por objetivo verificar a associação dos fatores associados às quedas em idosos de Estação, Rio Grande do Sul.

\section{MÉTODOS}

Trata-se de um estudo transversal de base populacional com idosos residentes no município de Estação, estado do Rio Grande do Sul. Estação encontra-se na região Noroeste do estado, distante 256,17 km da capital, Porto Alegre. Possuía, no momento da coleta de dados, uma população de 6.253 habitantes, dos quais 992 com idade igual ou superior a 60 anos, representando $15,9 \%$ da população total.
A Secretaria Municipal da Saúde de Estação dispunha de um ambulatório, que atuava na prestação de assistência básica à saúde da população. Essa unidade ambulatorial abriga a Estratégia da Saúde da Família (ESF), com cobertura de 100\% (cem por cento) de suas microáreas, um hospital de pequeno porte com fins lucrativos que mantém convênio com o município. A cidade referência em serviços de saúde para o município, que exigem infraestrutura com maior tecnologia, está localizada a uma distância de $31 \mathrm{~km}$. Na prestação de serviços assistenciais, dispõem-se de um médico de clínica geral, dois médicos de ESF, um médico pediatra, um médico ginecologista, três cirurgiões-dentistas, dois enfermeiros, dois técnicos em enfermagem, três auxiliares de enfermagem, um psicólogo e doze agentes comunitários de saúde.

Nesse contexto, o município realiza a terceirização de alguns serviços de exames laboratoriais, procedimentos e consultas médicas, procurando atender à demanda de seus munícipes por essas necessidades. Estação possui, ainda, convênio com municípios considerados referência no atendimento de urgência e emergência.

Para a identificação e localização da população do estudo, utilizou-se o Sistema de Informação da Atenção Básica (SIAB) da Secretaria de Saúde do município para o ano de 2011. Para o cálculo amostral, o erro aceitável adotado foi de 0,05 e intervalo de confiança de $95 \%$, resultando em 418 idosos. Considerando possíveis perdas de $10 \%$ (não elegibilidade, recusas, entre outras), um número adicional de idosos foi incluído como margem de segurança.

Conforme os dados apresentados pela Secretaria Municipal de Saúde, havia 992 idosos residentes em 12 microáreas de saúde no município de Estação-RS. Destas, oito são localizadas na zona urbana, com um total de 654 idosos; duas na zona rural, com 155 idosos; e duas estão distribuídas de forma mista (parte está localizada na zona urbana e parte na zona rural), com 183 idosos. Para a seleção dos participantes, com base nos registros da ESF levou-se em consideração a proporcionalidade da população investigada por sexo e zona de residência: $57 \%$ do sexo feminino e $43 \%$ do sexo masculino, $66 \%$ vivem na zona urbana, $16 \%$ na zona rural e $18 \%$ na zona mista.

Para cálculo do tamanho mínimo da amostra, adotou-se a Fórmula (1) segundo Calegari ${ }^{14}$, sendo:

$$
\mathrm{A}=\frac{\mathrm{N} \cdot \frac{1}{\mathrm{E}^{2}}}{\mathrm{~N}+\frac{1}{\mathrm{E}^{2}}}
$$

A seguir, foram selecionados por amostragem aleatória, mantendo as proporções estipuladas pela amostra. Portanto, incluíram-se no estudo 277 idosos da zona urbana, desses 158 do sexo feminino, 119 do masculino; na zona rural 67 idosos, desses 38 do sexo feminino e 29 do masculino; na zona mista 76 idosos, desses 43 do sexo feminino, 33 do masculino. 
Os critérios de inclusão foram: residir há pelo menos seis meses no território do município; possuir no ato da entrevista condições para responder ao questionário ou a presença de um familiar ou cuidador para auxiliar ou efetuar as respostas, não apresentar distúrbios psicológicos como: transtorno de comportamento e agressividade, não ter déficit cognitivo severo, não apresentar limitações físicas que comprometessem o deambular e aceitar participar do estudo. Consideraram-se como perdas os indivíduos que não foram localizados após três tentativas, em horários diferentes, e ao constatar com a vizinhança a ausência eram substituídos; mudança de residência para outro município; por óbito no período da coleta; indivíduos elegíveis que se recusaram a participar. Foram excluídos os idosos com dificuldades de comunicação que não tivessem um cuidador ou responsável para auxiliar na entrevista.

Coletaram-se os dados por meio de inquérito domiciliar, utilizando-se o questionário da pesquisa "Saúde, Bem-estar e Envelhecimento" - $\mathrm{SABE}^{15}$. Instrumento esse validado no Brasil para pessoas com 60 anos ou mais, de fácil aplicabilidade e fidedignidade ${ }^{15}$. O instrumento completo inclui sete seções: A) Informações pessoais e familiares, B) Avaliação cognitiva, C) Condições de moradia, D) Condições de saúde e hábitos de vida, E) Avaliação funcional, F) Uso e acesso aos serviços de saúde e G) Apoio familiar e social.

Neste estudo analisaram-se as seções A (informações pessoais e familiares: sexo, faixa etária, cor, reside, zona de moradia, estado marital e aposentadoria) e D (condições de saúde e hábitos de vida, como: dor crônica, quedas no último ano, alimentar-se sozinho, banhar-se, deitar/levantar da cama, subir/descer escadas, artrite/artrose, dificuldade auditiva, dificuldade visual, hipertensão arterial, reumatismo, osteoporose e derrame/isquemia cerebral). As variáveis: dor crônica, dificuldade auditiva e dificuldade visual foram mensuradas a partir do autorrelato dos idosos, ou seja, da percepção de cada idoso.

Foi realizado um teste- piloto com 40 idosos para averiguar se as instruções estavam claras na aplicação do instrumento aos respondentes. Cabe ressaltar que o instrumento foi aplicado em forma de entrevista para que pudesse diminuir os possíveis erros, sempre realizado por um mesmo avaliador.

As variáveis independentes relacionadas às características sociodemográficas foram: sexo, faixa etária, cor, reside, estado marital e aposentadoria. Outros fatores possivelmente associados às quedas incluíram as variáveis: atividade física, zona moradia, escolaridade, medicamentos, alimentar-se, banhar-se, deitar/levantar da cama e subir/descer escadas, artrite/artrose, dor crônica, dificuldade auditiva, dificuldade visual, reumatismo, hipertensão arterial, osteoporose e derrame/isquemia cerebral.

O projeto desta pesquisa foi aprovado pelo Comitê de Ética em Pesquisa da Universidade de Passo Fundo sob o parecer № 017/2011, CAAE n ${ }^{\circ}$ 0281.0.398.000.11. A coleta de dados se deu conforme a resolução 196/96 do Conselho Nacional de Saúde.

Realizou-se análise descritiva e bivariada dos dados. Inicialmente foi realizada frequência relativa e absoluta para caracterizar a amostra. Testou-se a associação entre o desfecho e as variáveis independentes através do teste qui-quadrado e análises brutas e multivariadas mediante regressão de Poisson, estimando-se as razões de prevalência brutas e ajustadas, calculados os respectivos intervalos de confiança de 95\%, com nível de significância de 0,05. Entraram no modelo múltiplo todas as variáveis com p-valor $\leq 0,20$.

\section{RESULTADOS}

Participaram da pesquisa 418 idosos de ambos os sexos, tendo maior prevalência entre o sexo feminino com $56,8 \%$, idade de 60 a 69 anos 50,0\%, e 63,8\% dos idosos apresentaram pelo menos uma queda no último ano. A Tabela 1 apresenta as características da amostra associadas às quedas, com destaque para o sexo feminino, a faixa etária de 80 anos ou mais, a cor branca e ser solteiro ou viúvo com maior percentual de ocorrência de quedas e estatisticamente significativo $(\mathrm{p} \leq 0,050)$.

$\mathrm{Na}$ análise bruta a não realização de atividade física mostra-se inicialmente associada à queda, tal como o analfabetismo e a presença de dificuldades para as tarefas básicas da vida diária (alimentação, banho, deitar e levantar da cama, subir e descer escadas) (Tabela 2). Ainda, a análise bruta revela associação entre histórico de quedas e presença de dor crônica $(\mathrm{p}<0,001)$, osteoporose $(\mathrm{p}=0,002)$ e isquemia cerebral $(\mathrm{p}<0,001)$.

No modelo ajustado apresentado na Tabela 3 para os fatores associados às quedas, permaneceram significativas as variáveis: ser analfabeto, apresentar dor crônica e ter sofrido isquemia cerebral.

Tabela 1. Características da amostra associadas às quedas dos idosos de Estação, RS, Brasil, $2011(n=418)$

\begin{tabular}{|c|c|c|c|}
\hline \multirow{3}{*}{ Variáveis } & \multicolumn{2}{|c|}{ Quedas } & \multirow{3}{*}{$p^{*}$} \\
\hline & Sim & Não & \\
\hline & n (\%) & n (\%) & \\
\hline Sexo & & & 0,005 \\
\hline Feminino & $72(30,3)$ & $166(69,7)$ & \\
\hline Masculino & $33(18,3)$ & $147(81,7)$ & \\
\hline Faixa etária & & & 0,014 \\
\hline 60 a 69 anos & $45(21,4)$ & $165(78,6)$ & \\
\hline 70 a 79 anos & $39(24,8)$ & $118(75,2)$ & \\
\hline$>80$ anos & $21(41,2)$ & $30(58,8)$ & \\
\hline Cor & & & 0,045 \\
\hline Branca & $97(26,8)$ & $265(73,2)$ & \\
\hline Parda/Negra & $8(14,3)$ & $48(85,7)$ & \\
\hline Reside & & & 0,103 \\
\hline Sozinho & $21(33,3)$ & $42(66,7)$ & \\
\hline Acompanhado & $84(23,7)$ & $271(76,3)$ & \\
\hline Estado marital & & & 0,005 \\
\hline Casado & $61(21,2)$ & $227(78,8)$ & \\
\hline Solteiro/viúvo & $44(34,1)$ & $85(65,9)$ & \\
\hline Aposentadoria & & & 0,322 \\
\hline Não & $15(20,5)$ & $58(79,5)$ & \\
\hline Sim & $90(26,1)$ & $255(73,9)$ & \\
\hline
\end{tabular}


Tabela 2. Associação bruta entre os fatores associados às quedas nos idosos de Estação, RS, Brasil, $2011(n=418)$

\begin{tabular}{|c|c|c|c|c|}
\hline \multirow{3}{*}{ Variáveis } & \multicolumn{2}{|c|}{ Quedas } & \multirow{3}{*}{$\begin{array}{l}\text { RP bruta } \\
\text { (IC 95\%) }\end{array}$} & \multirow{3}{*}{$p^{*}$} \\
\hline & Sim & Não & & \\
\hline & n (\%) & n (\%) & & \\
\hline \multicolumn{5}{|l|}{ Atividade física } \\
\hline Sim & $44(18,7)$ & $191(81,3)$ & 1,00 & \\
\hline Não & $61(33,3)$ & $122(66,7)$ & $0,56(0,40-0,79)$ & 0,001 \\
\hline \multicolumn{5}{|l|}{ Zona moradia } \\
\hline Rural & $35(31,0)$ & $78(69,0)$ & 1,00 & \\
\hline Urbano & $70(23,0)$ & $235(77,0)$ & $0,74(0,53-1,04)$ & 0,087 \\
\hline \multicolumn{5}{|l|}{ Alimentar-se } \\
\hline Sem dificuldade & $92(23,2)$ & $304(76,8)$ & 1,00 & \\
\hline Com dificuldade & $13(59,1)$ & $9(40,9)$ & $2,54(1,72-3,76)$ & $<0,001$ \\
\hline \multicolumn{5}{|l|}{ Banhar-se } \\
\hline Sem dificuldade & $88(23,1)$ & $293(76,9)$ & 1,00 & \\
\hline Com dificuldade & $17(45,9)$ & $20(54,1)$ & $1,99(1,34-2,95)$ & 0,001 \\
\hline \multicolumn{5}{|l|}{ Deitar/levantar cama } \\
\hline Sem dificuldade & $82(22,3)$ & $285(77,7)$ & 1,00 & \\
\hline Com dificuldade & $23(45,1)$ & $28(54,9)$ & $2,02(1,41-2,89)$ & $<0,001$ \\
\hline \multicolumn{5}{|l|}{ Subir/descer escadas } \\
\hline Sem dificuldade & $52(19,0)$ & $222(81,0)$ & 1,00 & \\
\hline Com dificuldade & $53(36,8)$ & $91(63,2)$ & $1,94(1,40-2,68)$ & $<0,001$ \\
\hline \multicolumn{5}{|l|}{ Artrite/artrose } \\
\hline Não & $78(23,8)$ & $250(76,2)$ & 1,00 & \\
\hline Sim & $27(30,0)$ & $63(70,0)$ & $1,26(0,87-1,83)$ & 0,219 \\
\hline \multicolumn{5}{|l|}{ Dor crônica } \\
\hline Não & $26(13,8)$ & $163(86,2)$ & 1,00 & \\
\hline Sim & $79(34,8)$ & $148(65,2)$ & $2,53(1,70-3,77)$ & $<0,001$ \\
\hline \multicolumn{5}{|l|}{ Dificuldade auditiva } \\
\hline Não & $67(22,8)$ & $227(77,2)$ & 1,00 & \\
\hline Sim & $38(30,9)$ & $85(69,1)$ & $1,36(0,97-1,90)$ & 0,078 \\
\hline \multicolumn{5}{|l|}{ Dificuldade visual } \\
\hline Não & $24(28,2)$ & $61(71,8)$ & 1,00 & \\
\hline Sim & $81(24,4)$ & $251(75,6)$ & $0,86(0,59-1,27)$ & 0,461 \\
\hline \multicolumn{5}{|l|}{ Escolaridade } \\
\hline$>4$ anos & $48(20,3)$ & $188(79,7)$ & 1,00 & \\
\hline 1 a 3 anos & $30(25,9)$ & $86(74,1)$ & $1,27(0,85-1,89)$ & 0,237 \\
\hline Analfabeto & $27(41,5)$ & $38(58,5)$ & $2,04(1,39-3,00)$ & $<0,001$ \\
\hline \multicolumn{5}{|l|}{ Reumatismo } \\
\hline Não & $86(24,2)$ & $270(75,8)$ & 1,00 & \\
\hline Sim & $19(30,6)$ & $43(69,4)$ & $1,27(0,84-1,93)$ & 0,264 \\
\hline \multicolumn{5}{|l|}{ Hipertensão arterial } \\
\hline Não & $36(22,6)$ & $123(77,4)$ & 1,00 & \\
\hline Sim & $69(26,6)$ & $190(73,4)$ & $1,18(0,83-1,67)$ & 0,364 \\
\hline \multicolumn{5}{|l|}{ Medicamentos } \\
\hline Não & $24(23,1)$ & $80(76,9)$ & 1,00 & \\
\hline Sim & $81(25,8)$ & $233(74,2)$ & $1,12(0,75-1,66)$ & 0,583 \\
\hline \multicolumn{5}{|l|}{ Osteoporose } \\
\hline Não & $87(23,1)$ & $289(76,9)$ & 1,00 & \\
\hline Sim & $18(42,9)$ & $24(57,1)$ & $1,85(1,25-2,75)$ & 0,002 \\
\hline \multicolumn{5}{|l|}{ Isquemia cerebral } \\
\hline Não & $95(23,7)$ & $306(76,3)$ & 1,00 & \\
\hline Sim & $10(58,8)$ & $7(41,2)$ & $2,48(1,61-3,83)$ & $<0,001$ \\
\hline
\end{tabular}

${ }^{\star}$ p-valor obtido pelo teste de Wald da regressão de Poisson 
Tabela 3. Razões de prevalência (RP) ajustadas e intervalos de confiança (IC95\%) para os fatores associados às quedas nos idosos de Estação, RS, Brasil, 2011. $(n=418)$

\begin{tabular}{|c|c|c|}
\hline Variáveis & $\begin{array}{l}\text { RP ajustada } \\
\text { (IC } 95 \%)\end{array}$ & $p^{*}$ \\
\hline \multicolumn{3}{|l|}{ Atividade física } \\
\hline Sim & 1,00 & \\
\hline Não & $0,74(0,50-1,11)$ & 0,150 \\
\hline \multicolumn{3}{|l|}{ Zona moradia } \\
\hline Rural & 1,00 & \\
\hline Urbano & $0,88(0,61-1,27)$ & 0,495 \\
\hline \multicolumn{3}{|l|}{ Escolaridade } \\
\hline$>4$ anos & 1,00 & \\
\hline 1 a 3 anos & $1,19(0,78-1,79)$ & 0,416 \\
\hline Analfabeto & $1,67(1,10-2,53)$ & 0,016 \\
\hline \multicolumn{3}{|l|}{ Alimentar-se } \\
\hline Sem dificuldade & 1,00 & \\
\hline Com dificuldade & $1,99(0,93-4,26)$ & 0,077 \\
\hline \multicolumn{3}{|l|}{ Banhar-se } \\
\hline Sem dificuldade & 1,00 & \\
\hline Com dificuldade & $0,74(0,31-1,80)$ & 0,513 \\
\hline \multicolumn{3}{|l|}{$\begin{array}{l}\text { Deitar/levantar } \\
\text { cama }\end{array}$} \\
\hline Sem dificuldade & 1,00 & \\
\hline Com dificuldade & $1,21(0,62-2,35)$ & 0,582 \\
\hline \multicolumn{3}{|l|}{$\begin{array}{l}\text { Subir/descer } \\
\text { escadas }\end{array}$} \\
\hline Sem dificuldade & 1,00 & \\
\hline Com dificuldade & $1,38(0,90-2,13)$ & 0,145 \\
\hline \multicolumn{3}{|l|}{ Dor crônica } \\
\hline Não & 1,00 & \\
\hline Sim & $2,34(1,55-3,53)$ & $<0,001$ \\
\hline \multicolumn{3}{|l|}{$\begin{array}{l}\text { Dificuldade } \\
\text { auditiva }\end{array}$} \\
\hline Não & 1,00 & \\
\hline Sim & $1,17(0,83-1,65)$ & 0,363 \\
\hline \multicolumn{3}{|l|}{ Osteoporose } \\
\hline Não & 1,00 & \\
\hline Sim & $1,32(0,88-1,97)$ & 0,182 \\
\hline \multicolumn{3}{|l|}{ Isquemia cerebral } \\
\hline Não & 1,00 & \\
\hline Sim & $2,30(1,54-3,42)$ & $<0,001$ \\
\hline
\end{tabular}

*p-valor obtido pelo teste de Wald da regressão de Poisson

\section{DISCUSSÃO}

Diante dos resultados expostos no modelo ajustado para os fatores associados às quedas, a prevalência de queda de nosso estudo $(63,8 \%)$ vai ao encontro de pesquisa realizada no Nordeste brasileiro por Santos et al. ${ }^{16}$ com percentual de $53,6 \%$ e corrobora ainda com a prevalência de $54 \%$ encontrada em Cuiabá ${ }^{17}$. Em estudo que utilizou como instrumento de pesquisa o $\mathrm{SABE}$, a prevalência de quedas nos últimos 12 meses foi de $56,3 \%$, o que está de acordo com os dados encontrados em nosso estudo ${ }^{18}$.
Em relação à escolaridade, os idosos analfabetos apresentaram maior ocorrência de quedas, quando comparadas aos escolarizados. Em estudo realizado no Rio Grande do Sul, Pereira et al. ${ }^{19}$, ao avaliarem 6.751 idosos, com média de idade de 70,3 anos, constataram que os idosos com maior escolaridade, ou seja, com grau superior, $94,4 \%$ apresentaram-se sem risco de quedas. Os autores creem que a maior escolaridade está associada a uma tendência a melhor renda, consequentemente melhores condições de moradia e acesso à saúde.

Em diversos estudos que investigaram os fatores associados às quedas em idosos, a escolaridade não se mostrou estatisticamente significativa ${ }^{20-23}$. Isso nos leva a concordar, portanto, com a afirmação da Organização Mundial da Saúde, de que o risco de quedas está ligado às condições socioeconômicas desfavoráveis, tais como situações de pobreza, rendimentos incertos ou insuficientes para garantir as necessidades básicas e acesso dificultado aos serviços de saúde ${ }^{6}$, e não somente ao grau de escolaridade, como encontrado em nossos resultados, o que torna a questão muito mais complexa e abrangente do que a contagem de anos que o indivíduo frequentou a escola.

Não há na literatura uma ampla investigação sobre a associação entre dor e queda, conforme Cruz et al. ${ }^{18}$, que ao avaliarem o índice de quedas em 213 idosos de São Paulo, acima de 60 anos e com dor crônica há mais de um ano, identificaram uma prevalência de queda autorreferida pelos idosos nos últimos 12 meses de 56,29\% [IC95\%: 47,33-65,24]. Referiram queda 63,43\% dos idosos com dor intensa e 52,27\% daqueles com dor moderada $(\mathrm{p}=0,04)$. Nesse contexto, a incapacidade física provocada pela dor pode possuir múltiplas variações, dependendo da localização e intensidade desta dor, entre outras características, como interferir nas atividades básicas da vida diária ${ }^{24}$.

Segundo Morais et al. ${ }^{25}$, ao analisarem o risco de quedas em 37 idosos com acidente vascular cerebral, com média de idade de 70,6 anos, constataram que o histórico de quedas nos últimos 6 meses foi menor do que o presente estudo, com 40,5\%. E 54\% dos idosos não utilizavam dispositivo auxiliares de locomoção, ou seja, outro agravante para o risco de quedas foi a presença do acidente vascular cerebral, interferindo de forma negativa na mobilidade física dos idosos.

Os idosos que sofreram algum acidente vascular encefálico (AVE) apresentam maiores riscos de quedas, dados esses afirmados pela pesquisa de Costa et al. ${ }^{26}$ ao identificar o risco de quedas em todos os participantes da pesquisa de uma amostra de 73 idosos, com média de idade de 69,5 anos, baixo nível de escolaridade com 4,9 anos. Dentre os fatores de risco encontrados, aqueles percebidos em aproximadamente $50 \%$ ou mais dos participantes de ambos os sexos foram: força diminuída nas extremidades inferiores, mobilidade física prejudicada, dificuldades na marcha, agentes anti-hipertensivos, equilíbrio 
prejudicado, idade acima de 65 anos e inibidores da enzima conversora angiotensina ${ }^{26}$.

Portanto, o risco de quedas decorrentes do processo de envelhecimento deve ser analisado como multifatorial, ou seja, diferentes fatores intrínsecos e extrínsecos podem vir a contribuir na ocorrência do número de quedas. Como exemplo pode se citar o risco de quedas em idosos que sofreram AVE, que pode chegar 102 vezes maior quando comparado aos idosos não acometidos por essa doença. Risco este ocasionado pelo equilíbrio prejudicado, decorrente da fraqueza muscular e espasticidade que dificultam a coordenação dos movimentos ${ }^{27}$.

Este estudo apresenta aspectos positivos como: um método rápido, prático e de baixo custo para testar o efeito e interação de um grande número de fatores que se relacionam com o evento estudado, neste caso a queda. Sugere-se que sejam desenvolvidos estudos com delineamento que permite estimar a incidência dos novos casos de quedas e seus motivos, ou seja, a cronologia nítida entre a exposição e o aparecimento do fator de risco que pode interferir na queda.

\section{CONSIDERAÇÕES FINAIS}

A prevalência de quedas entre idosos apresentou percentuais alarmantes, bem como demonstrado por diversos estudos nacionais, o que confere à queda entre os idosos um tema de discussão no âmbito da saúde pública.

Dentre os fatores relacionados às quedas, identificaram-se associação com o analfabetismo, às dores crônicas e os casos de isquemia cerebral. A investigação demonstra que os fatores associados às quedas são modificáveis e evitáveis. Atitudes voltadas à educação, saúde e prevenção podem interferir positivamente e reduzir as chances de quedas.

Dessa forma, recomenda-se que outros estudos de base populacional e com métodos mais complexos possam ser executados a fim de obter parâmetros sobre os fatores associados às quedas da população idosa brasileira em geral, para que se possa aplicar e criar programas de prevenção e educação em saúde que busquem o envelhecimento saudável.

\section{REFERÊNCIAS}

1. Williams JS, Kowal P, Hestekin H, O’Driscoll T, Peltzer K, Yawson A, et al. Prevalence, risk factors and disability associated with fall-related injury in older adults in low- and middle-incomecountries: results from the WHO Study on global AGEing and adult health (SAGE). BMC Med. 2015;2015(13):147. http://dx.doi.org/10.1186/s12916-015-0390-8. PMid:26099794.

2. Mesquita LS, Carvalho FT, Freire LS, Pinto O No, Zângaro RA. Effects of two exercise protocols on postural balance of elderly women: a randomized controlled trial. BMC Geriatr. 2015;2015(15):61. http://dx.doi.org/10.1186/ s12877-015-0059-3. PMid:26033080.

3. Karlsson MK, Magnusson H, Von Schewelov T, Rosengren BE. Prevention of falls in the elderly - a review. Osteoporos Int. 2013;24(3):747-62. http:// dx.doi.org/10.1007/s00198-012-2256-7. PMid:23296743.

4. Fang X, Shi J, Song X, Mitnitski A, Tang Z, Wang C, et al. Frailty in relation to the risk of falls, fractures, and mortality in older chinese adults: results from the Beijing Longitudinal Study of Aging. J Nutr Health Aging. 2012;16(10):903-7. http://dx.doi.org/10.1007/s12603-012-0368-6. PMid:23208030.

5. Jagnoor J, Keay L, Jaswal N, Kaur M, Ivers R. A qualitative study on the perceptions of preventing falls as a health priority among older people in Northern India. Inj Prev. 2014;20(1):29-34. http://dx.doi.org/10.1136/ injuryprev-2012-040707. PMid:23800638.

6. World Health Organization. Ageing: life course unit - WHO global report on falls prevention in older age [Internet]. France: WHO Library; 2007 [citado em 2016 abr 23]. Disponível em: http://www.who.int/ageing/ publications/Falls_prevention7March.pdf

7. Buksman S, Vilela ALS, Pereira SEM, Lino VS, Santos VH. Quedas em idosos: prevenção. São Paulo: Associação Médica Brasileira, Conselho Federal de Medicina, Sociedade Brasileira de Geriatria e Gerontologia; 2008. (Projeto Diretrizes).

8. Brasil. Ministério da Saúde. Anexo 01: protocolo prevenção de quedas. Brasília: Anvisa/Fiocruz; 2013
9. Damián J, Pastor-Barriuso R, Valderrama-Gama E, Pedro-Cuesta J. Factors associated with falls among older adults living in institutions. BMC Geriatr. 2013;13:6. PMid:23320746.

10. Grundstrom AC, Guse CE, Layde PM. Risk factors for falls and fallrelated injuries in adults 85 years of age and older. Arch Gerontol Geriatr. 2012;54(3):421-8. http://dx.doi.org/10.1016/j.archger.2011.06.008. PMid:21862143.

11. Hoops ML, Rosenblatt NJ, Hurt CP, Crenshaw J, Grabiner MD. Does lower extremity osteoarthritis exacerbate risk factors for falls in older adults? Womens Health (Lond Engl). 2012;8(6):685-98. http://dx.doi.org/10.2217/ WHE.12.53. PMid:23181533.

12. Ungar A, Rafanelli M, Iacomelli I, Brunetti MA, Ceccofiglio A, Tesi F, et al. Fall prevention in the elderly. Clin Cases Miner Bone Metab. 2013;10(2):915. PMid:24133524.

13. Recanello CG, Reiners AAO, Azevedo RCS, Alexandre RMS, Cegati L. Repercussões das quedas na vida dos idosos e seus familiares. Rev Enferm UFPE. 2015;9(3):7111-7.

14. Calegari JSM. Bioestatística: princípios e aplicações. Porto Alegre: Artemed; 2003.

15. Lebrão ML, Laurenti R. Saúde, bem-estar e envelhecimento: o estudo SABE no município de São Paulo. Rev Bras Epid. 2005;8(2):127-41. http://dx.doi. org/10.1590/S1415-790X2005000200005.

16. Santos RKM, Maciel ACC, Britto HMJS, Lima JCC, Souza TO. Prevalência e fatores associados ao risco de quedas em idosos adscritos a uma Unidade Básica de Saúde do município de Natal, RN, Brasil. Ciênc Saúde Coletiva. 2015;20(12):3753-62. http://dx.doi.org/10.1590/1413812320152012.00662015 .

17. Soares WJS, Moraes AS, Ferriolli R, Perracini MR. Fatores associados a quedas e quedas recorrentes em idosos: estudo de base populacional. 
Rev Bras Geriatr Gerontol. 2014;17(1):49-60. http://dx.doi.org/10.1590/ S1809-98232014000100006

18. Cruz HMF, Pimenta CAM, Dellarozza MSG, Braga PE, Lebrão ML, Duarte YAO. Quedas em idosos com dor crônica: prevalência e fatores associados. Rev Dor. 2011;12(2):108-14. http://dx.doi.org/10.1590/S180600132011000200006 .

19. Pereira GN, Morsch P, Lopes DGC, Trevisan MD, Ribeiro A, Navarro $\mathrm{JHN}$, et al. Fatores socioambientais associados à ocorrência de quedas em idosos. Ciênc Saúde Coletiva. 2013;18(12):3507-14. http://dx.doi. org/10.1590/S1413-81232013001200007.

20. Siqueira FV, Facchini LA, Piccini RX, Tomasi E, Thumé E, Silveira DS, et al. Prevalência de quedas em idosos e fatores associados. Rev Saude Publica. 2007;41(5):749-56. http://dx.doi.org/10.1590/S0034-89102007000500009. PMid:17923896.

21. Motta LB, Aguiar AC, Coutinho ESF, Huf G. Prevalência e fatores associados quedas em idosos em um município do Rio de Janeiro. Rev Bras Geriatr Gerontol. 2010;13(1):83-91. http://dx.doi.org/10.1590/S180998232010000100009 .

22. Ricci NA, Gonçalves DFF, Coimbra IB, Coimbra AMV. Fatores associados ao histórico de quedas de idosos assistidos pelo Programa de Saúde da Família. Saude Soc. 2010;19(4):898-909. http://dx.doi.org/10.1590/S010412902010000400016.
23. Chianca TCM, Andrade CR, Albuquerque J, Wenceslau LCC, Tadeu LFR, Macieira TGR, et al. Prevalência de quedas em idosos cadastrados em um Centro de Saúde de Belo Horizonte-MG. Rev Bras Enferm. 2013;66(2):23440. http://dx.doi.org/10.1590/S0034-71672013000200013.

24. Dellaroza MSG, Pimenta CAM, Duarte YA, Lebrão ML. Dor crônica em idosos residentes em São Paulo, Brasil: prevalência, características e associação com capacidade funcional e mobilidade (Estudo SABE). Cad Saude Publica. 2013;29(2):325-34. http://dx.doi.org/10.1590/S0102-311X2013000200019. PMid:23459818.

25. Morais HC, Holanda GF, Oliveira AR, Costa AG, Ximenes CM, Araujo TL. Identificação do diagnóstico de enfermagem "risco de quedas em idosos com acidente vascular cerebral”. Rev Gaúcha Enferm. 2012;33(2):117-24. http://dx.doi.org/10.1590/S1983-14472012000200017. PMid:23155589.

26. Costa AGS, Oliveira ARS, Moreira RP, Cavalcante TF, Araújo TL. Identificação do risco de quedas em idosos após acidente vascular encefálico. Esc Anna Nery. 2010;14(4):684-9. http://dx.doi.org/10.1590/S1414-81452010000400005.

27. Tanaka AFD, Scheicher ME. Relação entre depressão e desequilíbrio postural em idosos que sofreram acidente vascular encefálico. Fisioter Mov. 2013;26(2):315-20. http://dx.doi.org/10.1590/S0103-51502013000200008.

Recebido em: Out. 18, 2017 Aprovado em: Fev. 16, 2018 